\title{
Advanced Energy Efficient Passive Clustering Mobility in Wireless Sensor Networks
}

\author{
Aouad Siham, Maach Abdelillah and El Ganami Driss \\ Laboratory Networks and Intelligent Systems, \\ Mohammedia School of Engineering, Univeristy Mohammed V Agdal Rabat, Morocco
}

Received 2013-09-30, Revised 2013-10-02; Accepted 2013-10-24

\begin{abstract}
Wireless sensor networks have a wide applicability and increase deployment in the near future. In most of those applications, the network is composed of an important number of nodes deployed in an extensive area in which not all nodes are directly connected and based on clustering techniques. The Clustering can considerably reduce transmission overhead during flooding. In fact, by using clustering, we minimize the set of forwarding nodes during transmission and consequently reduce the energy cost and traffic overhead in topology environments. In this study, we present passive clustering mechanisms and the main clustering protocols proposed for wireless sensor networks; we also introduce a new protocol designated for mobile nodes in wireless sensor network that is based on the APC-T. This mechanism provides the stability of clusters after each departs of cluster-head and allows balanced energy consumption among the sensor nodes. Comparison with the existing schemes such as APC-T and Geographically Repulsive Insomnious Distributed Sensors (GRIDS) proves that the mechanism for selecting a backup of cluster-head nodes, which is the most important factor influencing the clustering performance, can significantly improves the network lifetime.
\end{abstract}

Keywords: Wireless Sensor Networks, Clustering Algorithms, Self-Organization, Energy Efficiency

\section{INTRODUCTION}

Recent advances in wireless communication and electronics have enabled the development of low cost, low power and energy-constrained sensors responsible for monitoring a physical phenomenon. This tiny system, which consists of a large number of sensor nodes deployed over a geographical area to extract information in hostile environments are known as wireless sensor network. Each node in the network of wireless sensor can monitor its environment and communicate the information collected in one or more collection points.

Each sensor node senses environmental conditions such as temperature, pressure and light and sends the sensed data to a Base Station (BS) or to other sensors. It includes three basic components: A sensing subsystem for data acquisition from the physical surrounding environment, a processing subsystem for local data processing and storage and a wireless communication subsystem for data transmission to a central collection point (sink node or base station (Anastasi et al., 2013)).

Wireless sensor networks can be used in a wide range of potential applications such as national security, surveillance, health care, biological detection, environmental monitoring and many other applications where fixed infrastructure is not easily acquired. In order to realize the existing and potential applications for WSNs, sophisticated and extremely efficient self organization protocols are required.

A sensor network is composed of several nodes distributed geographically to form a network without established infrastructure (Fig. 1). With the large number of sensor nodes deployed in remote environments, the ability of individual sensor nodes to self-organize is vital.

Corresponding Author: Aouad Siham, Laboratory Networks and Intelligent Systems, Mohammedia School of Engineering, Univeristy Mohammed V Agdal Rabat, Morocco 


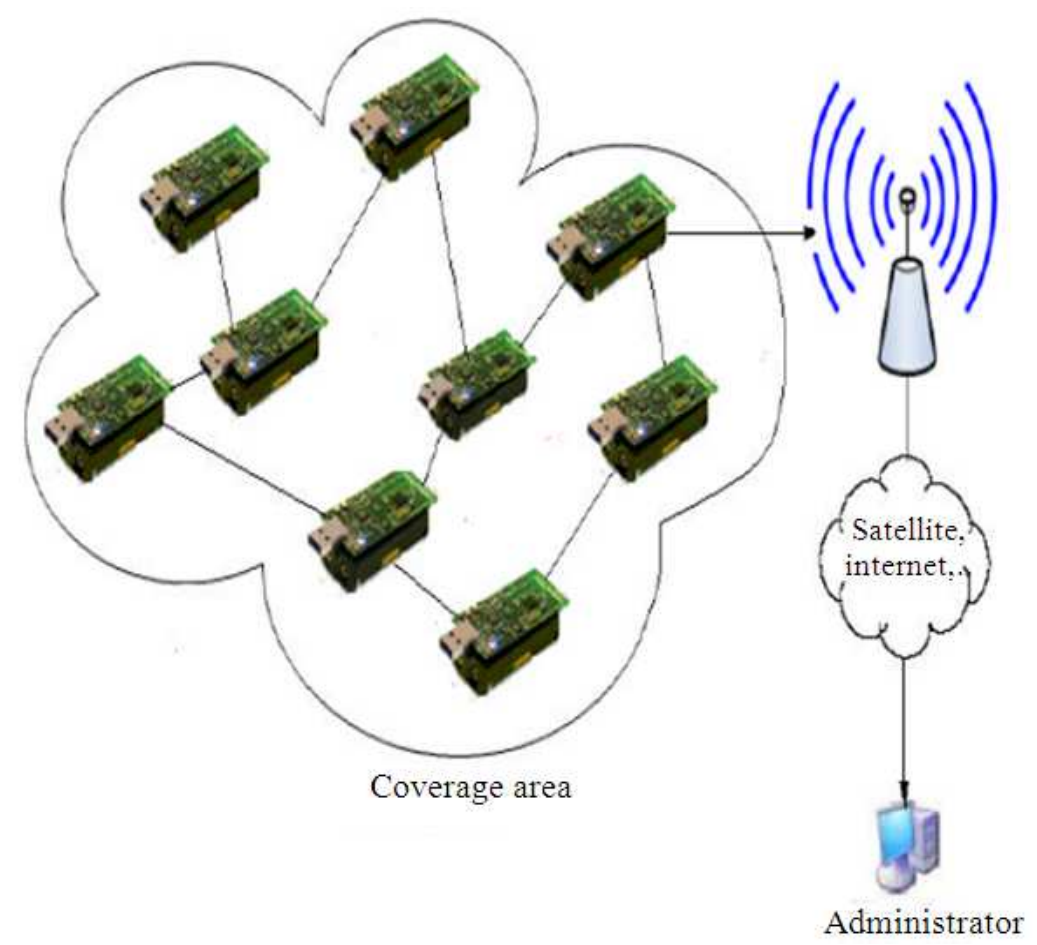

Fig. 1. Scheme of a wireless sensor network

The two main classes of self-organization protocols used today in wireless sensor networks are mainly based on clustering and multi-hop routing (see hybridization of two) Dehni et al. (2003).

Each sensor node is strongly power-constrained and one-off, the lifetime of WSN is limited. In order to extend the network lifetime, the research activities have been carried out to explore and overcome the constraints of WSNs and solve design and application issues. Efficiently organizing sensor nodes into clusters and elect one Cluster Head $(\mathrm{CH})$ for each cluster to transmit the collected data to central base station through other $\mathrm{CHs}$ is valuable in reducing energy consumption. Many energy-efficient protocols are designed based on the clustering structure Krishna et al. (1997) and McDonald and Znati (2001). The clustering technique can also used to perform data aggregation Mhatre et al. (2004) and Heinzelman et al. (2000). Obviously, we can save a great amount of unnecessary transmission by such clustering and data aggregation mechanisms.

Actually most of clustering approaches provide algorithms to balance energy consumption of network nodes, these techniques are basically turning the role of
$\mathrm{CH}$ randomly but it is not fault tolerant nodes especially nodes cluster-heads.

In this study, we propose an improved version of APC-T Aouad and Ganami (2012b) which enables a balanced energy consumption among the network nodes and extends the life time of the network by maintaining the cluster structure as long as possible.

This study is organized as follows. In the next, we outline the methods clustering and we describe our clustering algorithm AEEPC, its principle and its properties, after we present simulations and a discussion and at the end we introduce the perspectives.

\section{CLUSTERING MECHANISMS}

Clustering is a technique which allows assembling the nodes geographically close in groups called "clusters" and to establish routing scheme intracluster and inter-cluster. Clustering technique can be extremely effective in broadcast and data query $\mathrm{Ni}$ et al. (1999) and Estrin et al. (1999).

Each cluster is represented by a particular node called cluster-head. This node is elected by a specific metric or combination of metrics. The cluster-head will help to 
coordinate between the different members of the cluster, to collect and broadcast messages, to aggregate and sent the data collected to the base station.

Each node in a cluster stores all the information of its cluster and some of the information of other clusters; this minimizes considerably the size of routing tables and the number of messages exchanged in the network. So clustering gives the best results from the multi-hop routing, the reason we have also adopted this approach.

Clustering methods can be grouped into active, passive and hybrid ones. In active scheme, the synchronization between sensor nodes is necessary to construct and maintain the clusters. Contrariwise passive mechanism does not use any specific protocol control packets or signals. It exploits the data packets to transmit neighbor's information. Hybrid approaches use a combination of the above two methods.

\subsection{Active Clustering}

Active clustering uses a method of periodic exchange of Hello packets to collect information on the network topology. The first Active clustering algorithms were based on specific criteria for the selection of cluster-head Lowest-ID, Baker and Ephremides (1981) uses the identifiers of nodes and the number of neighbors, Basu et al. (2001) uses the degree of mobility, for against Low Energy Adaptive Clustering Hierarchy (LEACH) randomly selects cluster-heads nodes and assigns this role to different nodes according to the policy management RoundRobin (it means tourniquet) to ensure a fair energy dissipation between the nodes (Bagrodia et al., 1998).

These algorithms require two phases: Neighbor discovery and cluster formation phase. However, nodes are assumed fixed over the steps and synchronization between them is necessary for the success of these algorithms. In addition, following each change of network topology these steps are repeated periodically which degrades the stability of clusters.

\subsection{Passive Clustering $P C$}

Passive clustering Gerla et al. (2000) can be considered as on demand cluster formation protocol that does not use any protocol-specific control packets or signals. It allows exploiting the data packets to transmit neighbor's information.

The formation of cluster is automatic and initiated by the first data message to be flooded. By cons the clustering reduces the duration of the initial set-up period and the benefits of the reduction of the forwarding set can be felt by calculating the total energy consumed because the main function of the clusters is to optimize the exchange of flooded messages.

Passive Clustering uses the MAC frame (layer 2 of the $\mathrm{TCP} / \mathrm{IP}$ ) to encode the state of a network node; in particular, Passive Clustering uses two bits to encode 4 states.

(1) Initial, (2) Cluster head, (3) Gateway and (4) Ordinary. At the beginning, every sensor node is in the INITIAL state until it receives a packet. If the sender's packet is not CLUSTERHEAD, this sensor node changes state to CLUSTERHEAD-READY. This node wills bascule to state CLUSTERHEAD if it successfully transmits a packet before receiving any packets from others. If the sensor node receives a packet from a CLUSTERHEAD it switches state to ORDINADRY. Any sensor node that receives more than one message CLUSTERHEAD becomes GATEWAY.

Passive clustering is based in several mechanisms for the cluster formation such as: Gateway Selection Heuristic and First Declaration Wins algorithm. The Gateway Selection Heuristic allowed a method to elect the minimal number of gateways necessary to maintain the connectivity between cluster-heads. With the First Declaration Wins algorithm, a node that first forwards a data message will be a cluster-head and then it broadcasts its status to all its neighboring nodes.

With PC, each time a packet arrives at any node, the node needs only the state that is pasted in the received packet to the treatments for the selection of gateway, cluster-head or ordinary node. Only clusterhead and gateways that broadcast data packets and control; ordinary nodes receive the packet but do not participate in their broadcasts, Reducing collisions and reduces the amount of packets exchanged; therefore, there is a considerable decrease in energy consumption by the ordinary nodes which can increase the lifespan of WSN.

\subsection{Comparison between Active and Passive Clustering}

In Active clustering, aggregation of data is centralized and carried out periodically. However, in some cases, the periodic transmission of data may not be necessary, which rapidly depletes the limited energy of sensors. Passive clustering was defined to overcome/eliminate the limitations of active clustering in terms of overhead and energy consumption (more traffic means more energy consumption). So PC has many advantages compared at active clustering: 
- It reduces the latency of the formation of clusters and the additional traffic control by exploitation of data packets

- It uses a very effective heuristic that reduces the number of gateway during the delivery of packet

- It reduces energy consumption by eliminating the periodic sending of control information

- It is easy to imp lement and fully distributed

- Passive clustering can form better clusters than any conventional clustering schemes based on weight (e.g., ID and degree). This is because it utilizes data traffic information most important to solutions, which are relatively resistant from logical isolation and lack of connectivity

- Clustering stability is one of the major benefits of passive clustering; another is convergence time

\subsection{Passive Clustering Algorithms}

\subsubsection{Energy Conserving Passive Clustering (ECPC) Algorithm}

Basagni (1999), an important technique has been proposed "Energy Conserving Passive Clustering (ECPC) algorithm" which takes account of both residual energy and distance for elected cluster-head and gateway. Also it reduces the problem of idle listening through periodic sleep and awake among the cluster members. This algorithm surpasses the Directed Diffusion (DD) Siddiqui and Prakash (2002) and Passive Clustering Directed Diffusion (PCDD) Takai et al. (1999) in terms of energy consumption and network lifetime. It also generates much less gateway nodes than PCDD algorithm. The mixture of the clusterhead is based on the higher residual energy with in the 1-hop neighbors and the distance to form better clusters. The gateway selection procedure takes account the residual energy of the node which wants declare itself as a gateway and average distance of cluster-head nodes within 1-hop.

\subsubsection{Geographically Repulsive Insomnious Distributed Sensors (GRIDS)}

GRIDS (El Ghanami et al., 2008) is an energy-aware cluster formation protocol which increase the lifespan of a sensor network by using an efficient selection mechanism of critical (or not) nodes. This mechanism allows balanced energy consumption among the sensor nodes without requiring additional overheads including additional signaling, time synchronization and global information. GRIDS is based on an energy model which delivers node's residual/remaining energy level in real time and selects critical nodes among energy rich nodes, eFDW and eGSH models are the basic ideas of this protocol. This information is piggybacked in the nodes packet header. Each sensor determines being insomnious or not based on its residual energy and the number of neighboring insomnious nodes and their energy level. An efficient flooding during each wake up period determines insomnious nodes in the network. GRIDS selects insomnious nodes well distributed in the sensor deployed area. GRIDS inherit PC for constructing and maintaining clusters.

The main differentiator is that a set of nodes in a cluster with higher energy levels have higher probability to become critical nodes, i.e., $\mathrm{CH}$ or $\mathrm{GW}$. In $\mathrm{PC}, \mathrm{CHs}$ keep their cluster status until there is a $\mathrm{CH}$ collision, i.e., the hop distance between two CHs becomes 1 and one of them resigns from $\mathrm{CH}$. In GRIDS, an energy abundant node can challenge $\mathrm{CH}$ and usurps the role. Even if there is a $\mathrm{CH}$ declaration, nodes can challenge when their energy levels are higher than the one of $\mathrm{CH}$. These nodes keep their cluster status even if they receive packets from the current $\mathrm{CH}$. GRIDS distributes "evenly" the duty cycles among sensor nodes and thus considerably increase the network's lifespan as the result.

\subsubsection{Advanced Passive Clustering (APC)}

Advanced Passive Clustering (APC) (Aouad and Ganami, 2012a) is a protocol based on the Passive Clustering (PC) that does not use any specific protocol control packets. It exploits the data packets to transmit neighbor's information. At startup, all nodes are in the initial state. A node changes its state only when it receives a packet from its neighbors. In APC the $\mathrm{CH}$ selects from its neighbors list a $\mathrm{CH}_{-}$Bakup, this is the node that has the highest energy among all its neighbors. Once the $\mathrm{CH}$ leaves the cluster, $\mathrm{CH}_{-}$Backup replaces the $\mathrm{CH}$ and chooses its $\mathrm{CH}$ _Backup from its neighbors list. So APC maintains the structure of the cluster even at the leasing of $\mathrm{CH}$, increases the lifetime of the network and reduces energy consumption.

\subsubsection{Advanced Passive Clustering-Threshold (APC-T)}

Similarly, considering the APC algorithm, a valuable extension (that includes the concept of energy threshold) is proposed in Aouad and Ganami 
(2012a). (APC-T) where the information is included in the packet and the energy level of nodes is taken into account in the data transmission. Once the cluster is formed, the $\mathrm{CH}$ will select its $\mathrm{CH}_{-}$Backup from its neighbors list like APC algorithm; this is the node that has the highest energy among all its neighbors. In APCT, if the $\mathrm{CH}$ leaves the cluster, or its energy is below a given threshold T, CH_Backup replaces the $\mathrm{CH}$ and chooses its $\mathrm{CH}_{-}$Backup from its neighbors list. Figure 2 describe APC-T algorithm.

By this way, APC-T maintains cluster longer than APC and allows balanced energy consumption between the nodes of network.

\subsection{Advanced Energy Efficient Passive Clustering (AEEPC)}

Advanced Energy Efficient Passive Clustering (AEEPC) is a valuable extension to APC-T algorithm that includes additional Clusterhead-Backup election criteria and improves reliability of the network and uses balanced energy consumption among network nodes.
Consequently, the network stability and reliability are preserved, the transmission delay is decreased and the life time of the network is considerably increased.

\subsubsection{Principle}

Our new clustering algorithm is a protocol for cluster formation and election of clusterhead_Backup of the clusterheas. We summarize the AEEPC algorithm as follow: There are six possible states: Dead, Initial, Ordinary, Clusterhead_ready, Custer-head, Gateway and Clusterhead-Backup.

At cold start, all nodes are in the "initial" state, a node that joins the network, also starts with the initial state. This state does not change as long as a node does not receive a packet from another node. If the sender is not clusterhead, its status is cluster Head_Ready.

The clusterhead-ready will be clusterhead, if it can transmit packets before receiving any packet of another cluster-head. If the packet comes from another clusterhead, the node records its id, the time of receipt and adds this node to the list of clusterhead and then it switches to Ordinary.

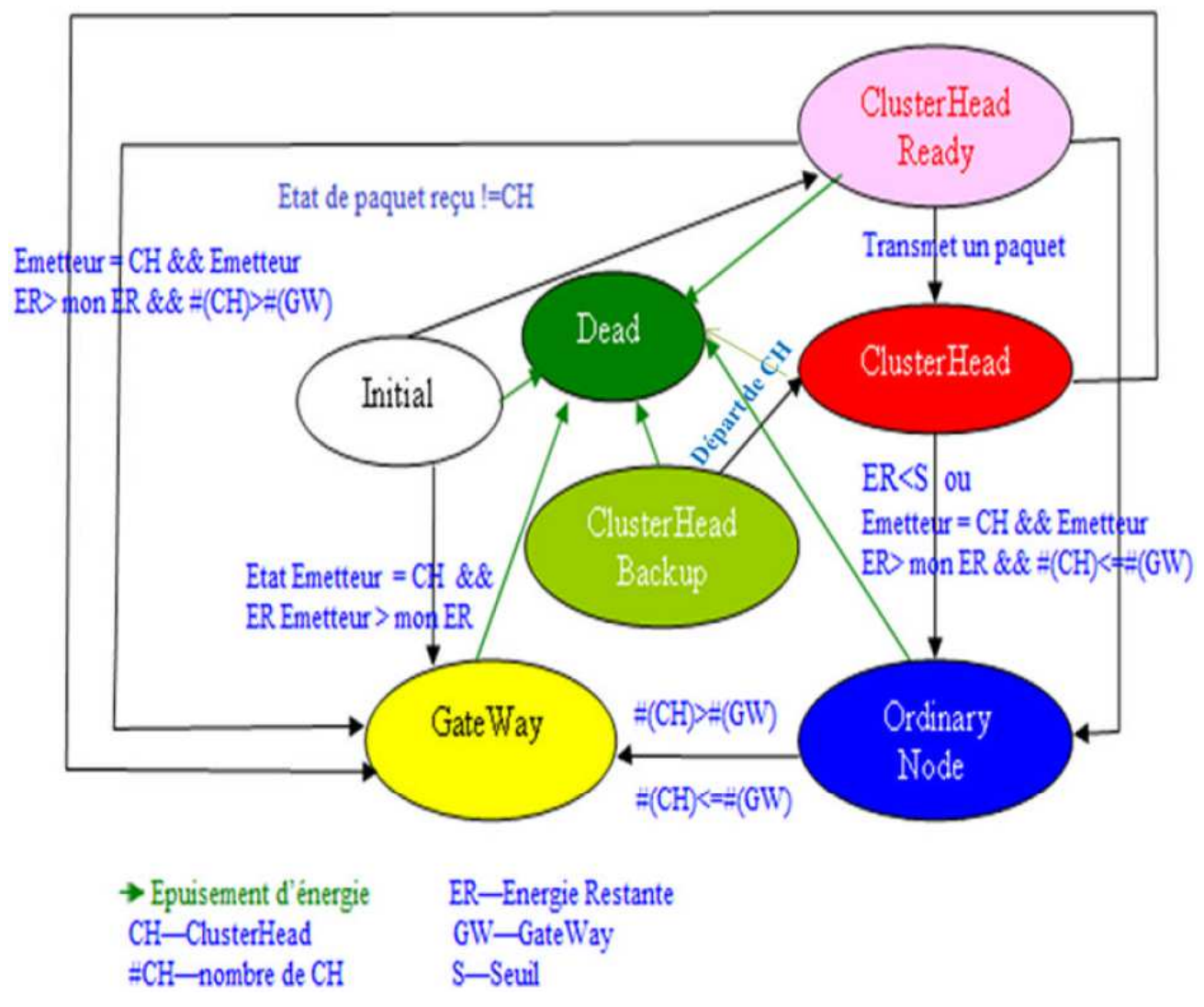

Fig. 2. APC-T automate 
The node ClusterHead_ready switches to state gateway when the number of ClusterHeads is greater or equal to the number of Gateways. Otherwise, the node becomes an Ordinary Node or an alternate node. The clusterhead selects from its neighbors list a ClusterHead_Bakup, this is the node that has the highest coefficient $\mathrm{K} \mathrm{n}$ among all its neighbors; with:

$$
\begin{array}{lll}
\mathrm{K}_{\mathrm{n}}= & \mathrm{a}_{1} \mathrm{E}_{\mathrm{n}}+\mathrm{a}_{2} \mathrm{D}_{\mathrm{n}} \\
\mathrm{E}_{\mathrm{n}}= & \mathrm{E}_{\text {remaining }}(\mathrm{n}) \div \mathrm{E}_{\text {Initial }}(\mathrm{n}) \\
\mathrm{D}_{\mathrm{n}}= & \text { (The average distance between the node } \mathrm{n} \\
& \text { with all other nodes in the same cluster } \div \\
& \text { (The maximum range of a node) } \\
\mathrm{a}_{1}+\mathrm{a}_{2}= & 1
\end{array}
$$

Once the cluster-head leaves the cluster, or its energy is below a given threshold T, ClusterHead_Backup replaces the clusterhead and launch the procedure to select a backup like in APC-T (Fig. 3). The first $\mathrm{CH}$ switches to ordinary state:

- An ordinary node switches to ClusterHead-Backup if its $\mathrm{Kn}$ is higher than the actual $\mathrm{CH}_{-}$Backup

- All non-clusterhead nodes maintain a neighbors list of clusterhead. When a node receives a packet from a clusterhead, it updates the list of clusterhead at the same time it checks the number of active clusterhead. If it is greater than or equal to two node becomes Gateway. In general, in mobile ad hoc networks and wireless sensors, the number of gateways is significant and can exceed the number of Ordinary nodes, so we must reduce the number of gateways for effective flood

- Several heuristics are used to elect a clusterhead in each cluster following a weight calculated that distinguishes it from other network nodes. As an example algorithm "c Mobility Adaptive Clustering" in Basagni (1999) and Siddiqui and Prakash (2002) introduced the notion of generic weights for the selection of clusterhead. A clusterhead, which does not receive a packet from gateway during a preset time, change its status to normal. This node broadcasts all packets that it receives

- Each node collects information about the neighbors. It stores id, status and idle time-if the idle time beyond the timeout threshold, the entry is deleted

Table 1 presents a pseudo-code that shows the operation details of AEEPC.

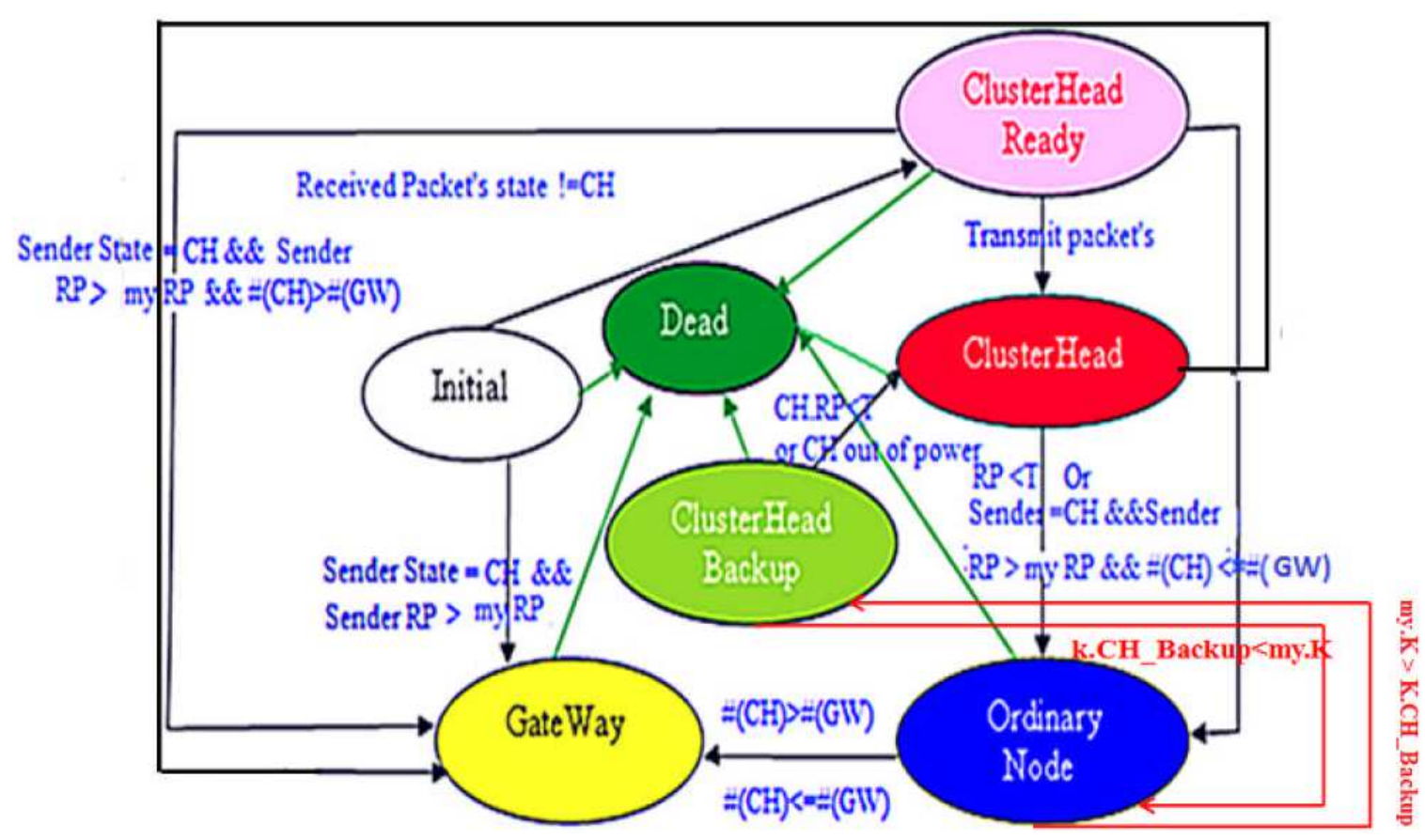

Fig. 3. AEEPC automat 
Table 1. Pseudo code of AEEPC

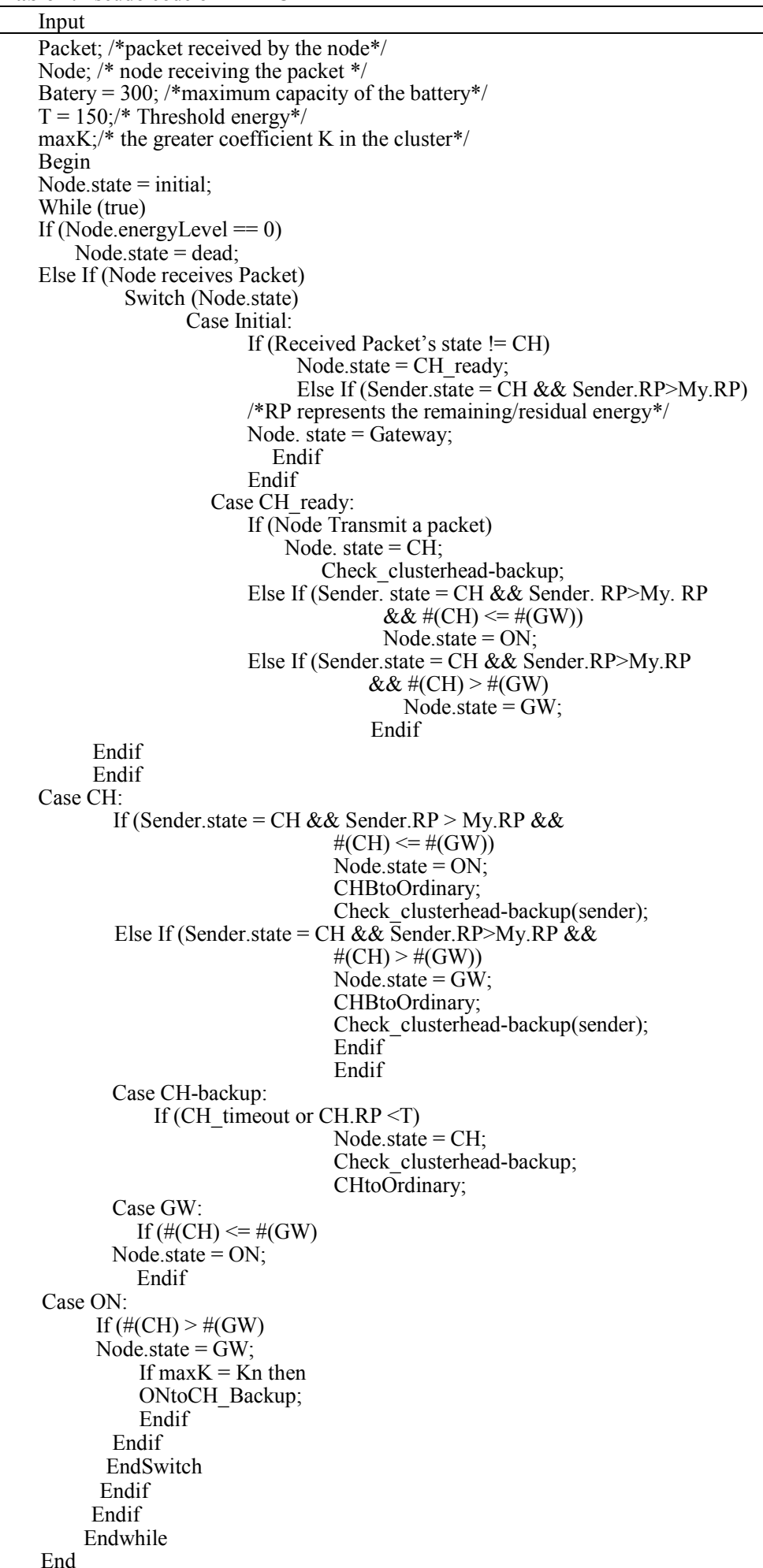




\section{SIMULATIONS AND RESULTS}

To evaluate the performance of the proposed clustering mechanism, we conducted a series of simulations and we compare aour algorithm with other two most significant clustering protocols, APC-T and GRIDS. The simulation models used were implemented in the GloMoSim library Takai et al. (1999), which is a scalable simulation environment for wireless networks based on the Parsec language Bagrodia et al. (1998). The radio propagation range for each node is 150 meters and channel capacity is 2 Mbits $\mathrm{sec}^{-1}$. The roaming space is $600 \times 600$ meters square. The size of the network is 300 nodes; randomly in the roaming area. The node's maximum speed is $4 \mathrm{~m} \mathrm{sec}^{-1}$. The Distributed Coordination Function (DCF) of IEEE 802.11 is used as the MAC layer. Each simulation is executed for $6 \mathrm{~min}$. Traffic sources are CBR. The source destination pairs are totally randomized. Data packets are all 512 bytes long. Control packet length is 32 bytes. The random waypoint model was used for node mobility. Each node sends 100 packets with inter-arrival time of 0.5 sec. We use Ad hoc On-demand Distance Vector routing (AODV) (Johnson, 1997) because AODV is one of the most flooding-dependent routing protocols.

The objective of the simulations is to show that the AEEPC maintains the structure of the cluster even at the leasing of clusterhead and thus increases the lifetime of the network and reduces energy consumption. We use three metrics for analyze and compare the simulation results: network lifetime, energy wasting and delivery ratio at base station.

Statistics Fig. 4 shows that the proposed algorithm and APC-T consume more energy for a number of nodes less than 400 nodes than GRIDS there's an additional load due to the election of clusterhead_backups. Byagainst, they consume less energy for a greater number of nodes than GRIDS and APC-T. Thus, we conclude that this algorithm is more suitable for large scale networks and allows balanced energy consumption among the network nodes. In this simulation we use the parameters of Table 2.

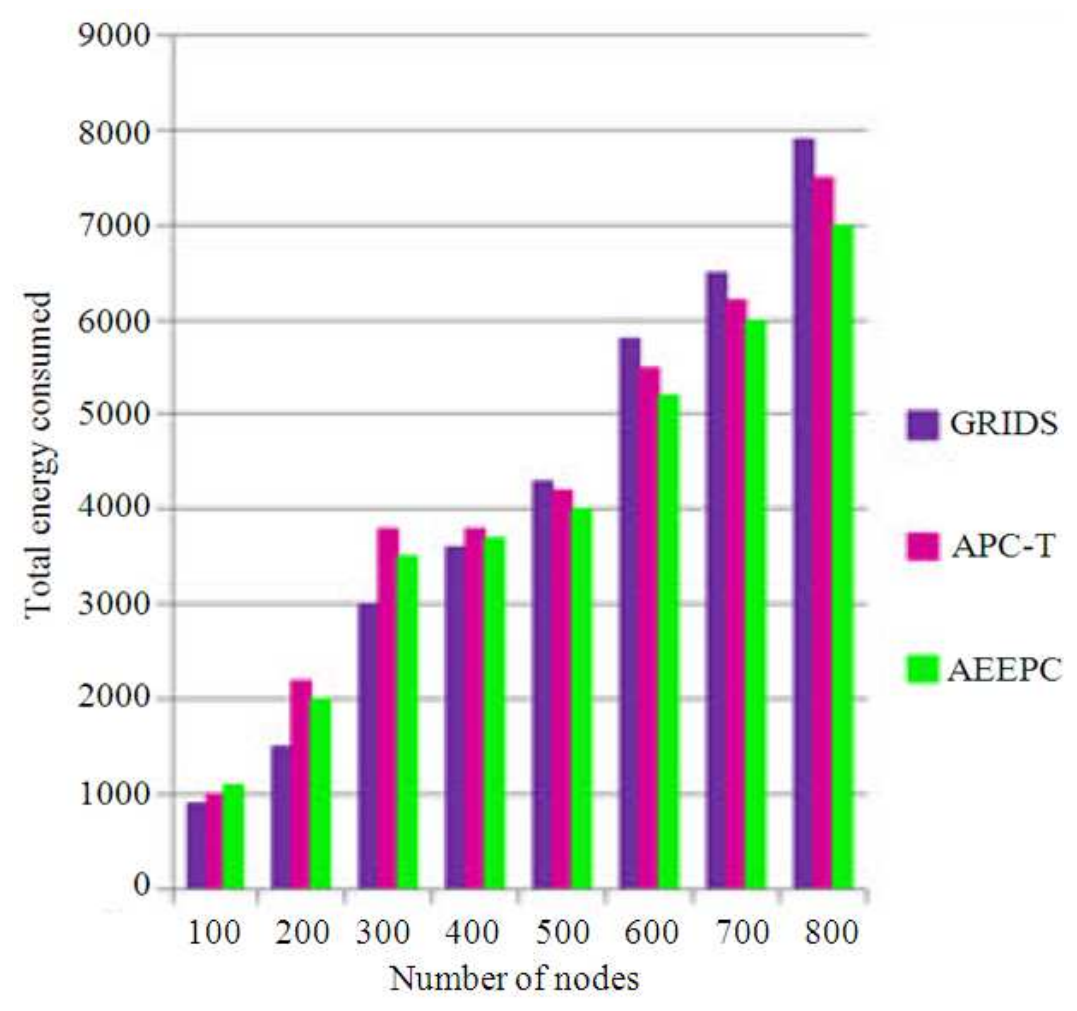

Fig. 4. Comparison of energy consumption 


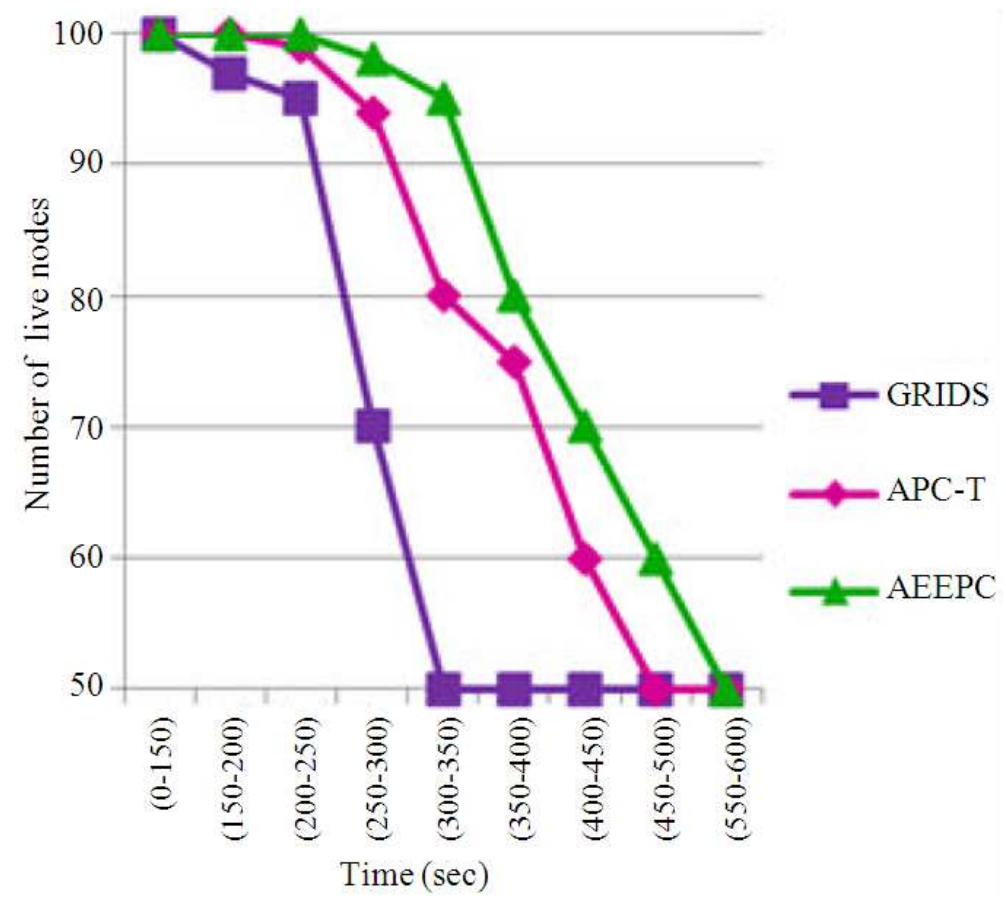

Fig. 5. Percentage of live nodes

Table 2. Simulation parameters for a mobile environment

\begin{tabular}{ll}
\hline Parameters & Values \\
\hline Movement speed & $\{2,4,6,8,10\} \mathrm{m} . \mathrm{s}-1$ \\
Number of nodes & 50 \\
Transmission range & $(\mathrm{Rt}) 20 \mathrm{~m}$ \\
Surface deployment & $100 \times 100 \mathrm{~m}$ \\
\hline
\end{tabular}

The percentage of active nodes gives an idea about the effectiveness of the algorithm; the higher percentage means that the network performance is more efficient. In this simulation, we assume that the network performs its tasks successfully while ensuring full coverage of the area of interest as a-coverage exceeds 50\% Lehsaini et al. (2008).

Figure 5 shows that the lifetime of the network is longer with AEEPC than GRIDS and APC-T. In GRIDS, the first sensor stops working in the time interval (300-350) while with APC-T and with APC-T it stops working in the time interval (450-500) and with AEEPC, it stops working after $50 \mathrm{sec}$. than APC$T$. This is due to balancing energy among the network and the concept of backup introduced. So AEEPC ensures more stability of network and reduces the number of isolated nodes and subsequently increases the lifetime of network.

In this simulation we estimate the average number of clusters produced according to the speed of movement of nodes. Therefore, we compared AEEPC with APC and GRIDS.

Among the evaluation of the effectiveness of a clustering algorithm, we find the number of clusters criteria. According to Chen and Stojmenovic (1999) as the number of clusters formed is reduced as the clustering algorithm is efficient.

In Fig. 6 we observed that the number of clusters formed by our algorithm is lower than one generated by the APC-T and GRIDS algorithm. Also AEEPC is fairly stable during all the simulation time. This stability is illustrated by the small variation in the number of clusters formed by this algorithm.

We noticed that the average of cluster-head (Fig. 7) change in AEEPC and APC-T are less than GRIDS algorithm and it is fairly stable in AEEPC algorithm. This stability is explained by the small variation of this average and to alternating state between $\mathrm{CH}$ and $\mathrm{CH}$ Backup, which maintain the cluster structure farther. So AEEPC increases the lifespan of the network and reduces energy dissipation. 
Aouad Siham et al. / American Journal of Applied Sciences 10 (12): 1558-1569, 2013

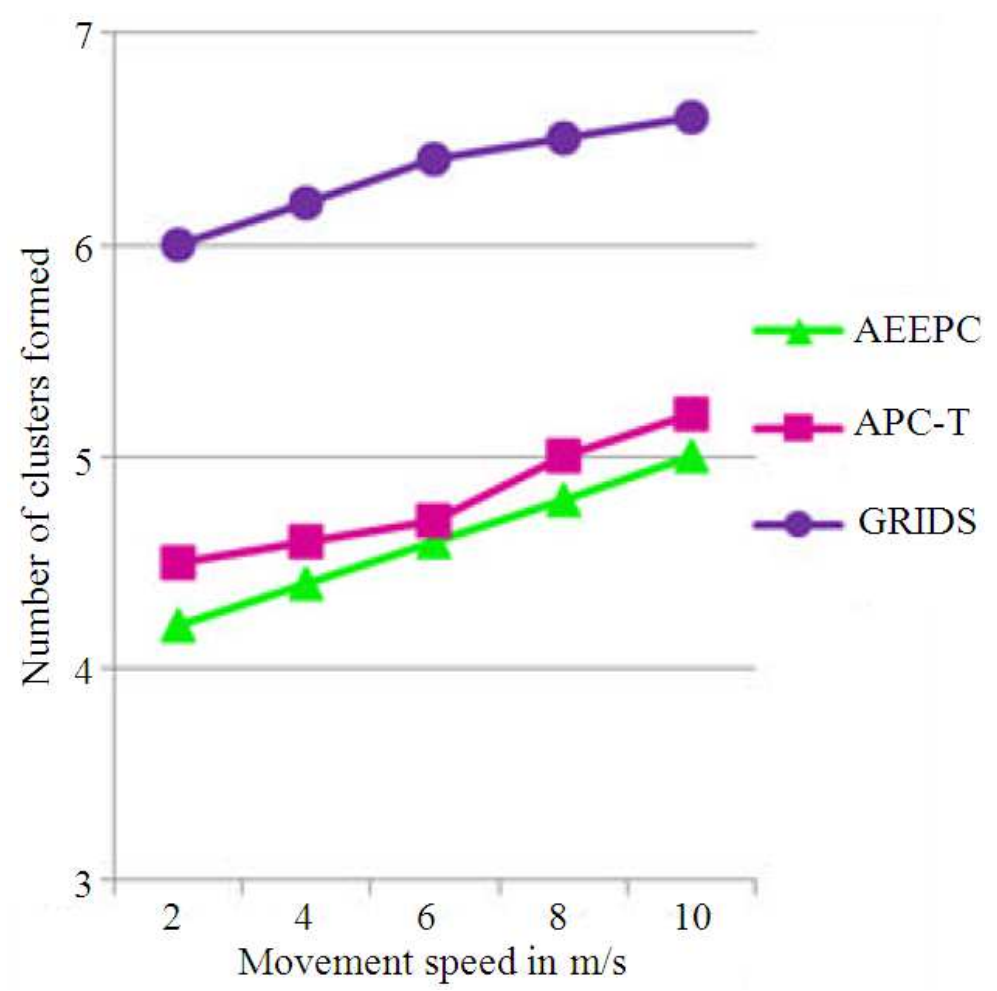

Fig. 6. Average of the clusters formed according to movement speed

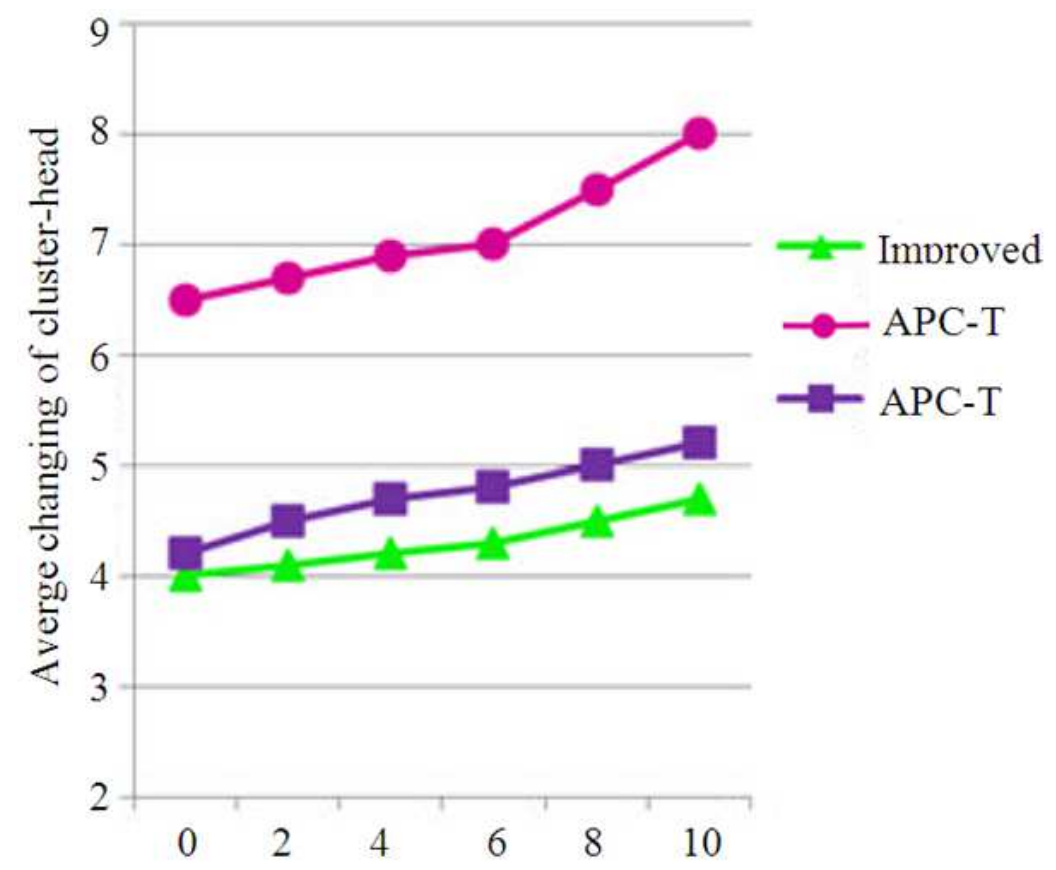

Fig. 7. Average of the cluster-head change depending on movement speed 


\section{DISCUSSION}

In this article, we proposed a new algorithm based on passive clustering. It represents a curative solution for mobility in wireless sensor networks. Simulation results show the effectiveness of the approach in reducing the amount of energy consumed by the network in comparison with two well-known protocols, GRIDS and APC-T.

\section{CONCLUSION}

This study introduces several contributions; first, the electing cluster-heads and Clusterhead_Backup in wireless sensor networks. The selection of Clusterhead_Backup is performed according to the remaining energy and the average distance between the nodes of the cluster. The second, the role of clusterheads and Cluster Head_Backups can be switched dynamically if the energy of cluster-head is below a given threshold $\mathrm{T}$ or its leaves the cluster. In the future, we plan to study different failure scenarios in sensor networks and introduce run-time faulttolerance in the system.

\section{REFERENCES}

Anastasi, G., M. Conti, M. Di Francesco and A. Passarella, 2013. How to Prolong the Lifetime of Wireless Sensor Networks. In: Mobile Ad Hoc and Pervasive Communications, Denko, M. and L. Yang (Eds.), CRC Press.

Aouad, S. and D. Ganami, 2012b. Advanced passive clustering-threshold a maintenance mechanism of cluster structure. J. Theoretical Applied Inform. Technol., 46: 0587-0593.

Aouad, S. and D.E. Ganami, 2012a. Advanced passive clustering. Proceedings of the International Conference on Multimedia Computing and Systems, May 10-12, IEEE Xplore Press, Tangier, pp: 468472. DOI: 10.1109/ICMCS.2012.6320150

Bagrodia, R., R. Meyer, M. Takai, Y. Chen and X. Zeng et al., 1998. Parsec: A parallel simulation environment for complex systems. Computer, 31: 77-85. DOI: 10.1109/2.722293

Baker, D.J. and A. Ephremides, 1981. The architectural organization of a mobile radio network via a distributed algorithm. IEEE Trans. Commun., 29: 1694-1701. DOI: 10.1109/TCOM.1981.1094909
Basagni, S., 1999. Distributed and mobility-adaptive clustering for multimedia support in multi-hop wireless networks. Proceedings of the IEEE VTS 50th Vehicular Technology Conference, Sept. 1922, IEEE Xplore Press, Amsterdam, pp: 889-893. DOI: 10.1109/VETECF.1999.798457

Basu, P., N. Khan and T.D.C. Little, 2001. A mobility based metric for clustering in mobile ad hoc networks. Proceedings of the 21st International Conference on Distributed Computing Systems, Apr. 16-19, Meas, AZ., pp: 413-418. DOI: 10.1109/CDCS.2001.9187738

Chen, G. and I.C. Stojmenovi, 1999. Clustering and routing in mobile wireless networks. Technical Report TR-99-05, University of Ottawa.

Dehni, L., Y.B. Eennabi and F. Krief, 2003. LEA2C: Une nouvelle approche de routage dans les réseaux de capteurs pour l'optimisation de la consummation d'ènergie. Université Paris 13.

El Ghanami, D., T.J. Kwon and A. Hafid, 2008. GRIDS: Geographically repulsive insomnious distributed sensors-an efficient node selection mechanism using passive clustering. WiMob, 2008: 241-246.

Estrin, D., R. Govindan, J. Heidemann and S. Kumar, 1999. Next century challenges: Scalable coordination in sensor networks. Proceeding of the 5th Annual ACM/IEEE International Conference on Mobile Computing and Networking, Aug. 15-19, Seattle, WA, USA., pp: 263-270. DOI: 10.1145/313451.313556

Gerla, M., T.J. Kwon and G. Pei, 2000. On-demand routing in large ad hoc wireless networks with passive clustering. Proceedings of the Wireless Communications and Network Conference, Sept. 23-28, IEEE Xplore Press, Chicago, IL., pp: 100105. DOI: 10.1109/WCNC.2000.904608

Heinzelman, W.R., A. Chandrakasan and H. Balakrishnan, 2000. Energy-efficient communication protocol for wireless microsensor networks. Proceedings of the 33rd Annual Hawaii International Conference on System Sciences, Jun. 4-5, IEEE Xplore Press, pp: 8020-8020. DOI: 10.1109/HICSS.2000.926982

Johnson, D.B., 1997. Routing in Ad Hoc networks of mobile hosts. Proceedings of the Workshop on Mobile Computing and Applications, Jun. 8-12, IEEE Xplore Press, Montreal, Que, pp: 376-380. DOI: 101109/ICC.1997.605303 
Krishna, P., N.H. Vaidya, M. Chatterjee and D. Pradhan, 1997. A cluster-based approach for routing in dynamic networks. ACM SIGCOMM Comput. Commun. Rev., 27: 49-65. DOI: 10.1145/263876.263885

Lehsaini, M., H. Guyennet and M. Feham, 2008. Acoverage scheme for wireless sensor networks. Proceedings of the 4th International Conference on Wireless and Mobile Communications, Jul. 27-Aug. 1, Athens, Greece, pp: 91-96. DOI: 10.1109/ICWMC.2008.67

McDonald, A.B. and T.F. Znati, 2001. Design and performance of a distributed dynamic clustering algorithm for ad-hoc networks. Proceedings of the Annual Simulation Symposium, Apr. 22-26, IEEE Xplore Press, Seattle, WA., pp: 27-35. DOI: 10.1109/SIMSYM.2001.922111

Mhatre, V., C. Rosenberg, D. Kofman, R. Mazumdar and N. Shroff et al., 2004. Design of surveillance sensor grids with a lifetime constraint. Proceedings of the 1st European Workshop on Wireless Sensor Networks Berlin, Jan. 19-21, Springer Berlin Heidelberg, Berlin, Germany, pp: 263-275. DOI: 10.1007/978-3-540-24606-0_18
Ni, S., Y. Tseng, Y. Chen and J. Sheu, 1999. The broadcast storm problem in a mobile ad hoc network. Proceedings of the Annual ACM/IEEE International Conference on Mobile Computing and Networking, Aug. 15-19, Seattle, WA, USA., pp: 151-162. DOI: 10.1145/313451.313525

Siddiqui, A.A. and R. Prakash, 2002. Effect of availability factor threshold and clustering gap on performance of clustering mechanisms for multicluster mobile ad hoc networks. Proceedings of the IEEE International Conference on Communications, Apr. 28-May 2, IEEE Xplore Press, pp: 3296-3300. DOI: 10.1109/ICC.2002.997 442

Takai, M., L. Bajaj, R. Ahuja, R. Bagrodia and M. Gerla, 1999. GloMoSim: A scalable network simulation environment. Department of Computer Science. 